\title{
Formamides as Isocyanate Surrogates: A Mechanistically-Driven Approach to the Development of Atom-Efficient, Selective Catalytic Syntheses of Ureas, Carbamates and Heterocycles
}

\author{
Jeffrey Bruffaerts, Niklas von Wolff, + Yael Diskin-Posner, Yehoshoa Ben-David, David Milstein* \\ Department of Organic Chemistry, The Weizmann Institute of Science, Rehovot 76100, Israel
}

Green Chemistry - Homogeneous Catalysis - Mechanistic Investigations - Computational Study - Pincer Complexes

\begin{abstract}
Despite the hazardous nature of isocyanates, they remain key building blocks in bulk and fine chemical synthesis. By surrogating them with less potent and readily available formamide precursors, we herein demonstrate an alternative, mechanistic approach to selectively access a broad range of ureas, carbamates and heterocycles via a ruthenium-based pincer complex catalyzed acceptorless dehydrogenative coupling reactions. The design of these highly atom-efficient procedures was driven by the identification and characterization of the relevant organometallic complexes, uniquely exhibiting the trapping of an isocyanate intermediate. DFT calculations further contributed to shed light on the remarkably orchestrated chain of catalytic events, involving metal-ligand cooperation.
\end{abstract}

\section{INTRODUCTION}

Due to environmental and safety concerns, there is an urgent need to render chemical synthesis not only more efficient, but also less hazardous for humans and the biosphere. ${ }^{1}$ These efforts have led to: $(i)$ the banning of notoriously harmful chemicals while finding sustainable alternatives; $^{2}$ (ii) and to significantly reduce the amounts of waste generated by fostering atom and step-efficient strategies. ${ }^{3-5}$ Although the environmental footprint of a given chemical process may be continually optimized, surrogating a key but harmful chemical reagent generally appears more challenging. For instance, isocyanates belong to this class of essential though notoriously sensitive and toxic compounds. Their industrial importance has continuously grown over the century, as isocyanates are inevitable precursors of urea and carbamate linkages found in polyurethanes, drugs, pesticides, etc (Scheme 1, A) ${ }^{6}$ The most-produced isocyanate, methylisocyanate (MIC), has been most (in)famously known since the Bhopal disaster, 7,8 tragically highlighting the hazards of stocking large amounts of volatile and toxic chemicals. Besides improving safety containments for such reagents, it would fundamentally be safer to substitute them with less potent -though still inexpensive- surrogates. To circumvent the direct usage of an isocyanate, an alternative approach consists in pre-activating amines or alcohols (typically with phosgene, or any other synthetic equivalent synthon). Yet, on large scale, such two-step strategies may suffer from the toxicity of phosgene (if employed) and the waste consequently generated (as additional bases are usually required). Assessing the issues of classical approaches to generate urea and carbamate bonds further stresses the importance of developing greener selective alternatives.

In the context of the modern green(r)evolution in chemical synthesis, the rise of catalysis has not only contributed to reduce the energetic and environmental impact of reactions, but also to reveal novel and unforeseen synthetic strategies. ${ }^{9}$ Specifically, the rational design of increasingly capable and sophisticated transition-metal based homogeneous complexes has considerably enhanced the potential of catalyzed reactions. For example, metal-ligand cooperation (MLC), inspired by Nature's cooperative enzymatic systems, has shown great promises in targeting covalent bond activation through active ligand participation. ${ }^{10}$ This key feature enables, in some cases, to significantly reduce covalent bond activation energies, thus enabling new synthetically useful catalytic reactions. Complexes functioning by metal-ligand cooperation have been especially useful in catalytic acceptorless dehydrogenative coupling reactions, typically allowing the $\alpha$-functionalization of alcohols or amines through in situ formation of aldehyde or imine intermediates, respectively. ${ }^{10-14}$ Advantageously, such transformations catalytically generate these reactive and sensitive intermediates while only releasing dihydrogen as by-product. This approach has led to the development of highly atom-efficient and straightforward transformations. The inherent advantages offered by this greener strategy prompted us to explore the potential of substituting isocyanates by formamides, considerably less noxious and more stable reagents (Scheme 1, B).

Interestingly, pioneering reports critically suggested the possibility of catalytically generating isocyanates from $\mathrm{N}$ arylformamides to access ureas ${ }^{15}$ and carbamates ${ }^{16}$ using ruthenium-based complexes, although yields and turn-over numbers were relatively modest. Only recently, this transformation has further drawn attention through the utilization of PNP-type pincer complexes based on ruthenium ${ }^{17,18}$ and iron, ${ }^{19}$ although limited to amine nucleophiles and without the possibility of surrogation of the notorious MIC. Dedicated to further promote and exploit the full potential of 
this remarkable and somewhat undervalued reaction, we developed an interest in fundamentally understanding its mechanism, essential to potentially address the current synthetic limitations. Indeed, the selectivity for the desired transformation can be drastically hampered by numerous (catalyzed or uncatalyzed) side reactions. For instance, we and others ${ }^{16-19}$ observed cases of (i) formamide decarbonylation, (ii) transamidation / esterification, (iii) competitive dehydrogenative pathways and (iv) urea scrambling
(Scheme 1, C) leading to limited substrate scope and selectivity. These numerous side reactions further highlight the remarkable challenge of finding the optimal experimental conditions to efficiently and selectively generate urea or carbamate moieties from formamides. To address the selectivity issues, we anticipated that insight into the mechanistic events at play may potentially empower us to rationally enhance its scope, as well as spark new opportunities.

Scheme 1. Approaches to access ureas and carbamates $(X=$ leaving group, $A l k=$ alkyl, $A r=a r y l)$.
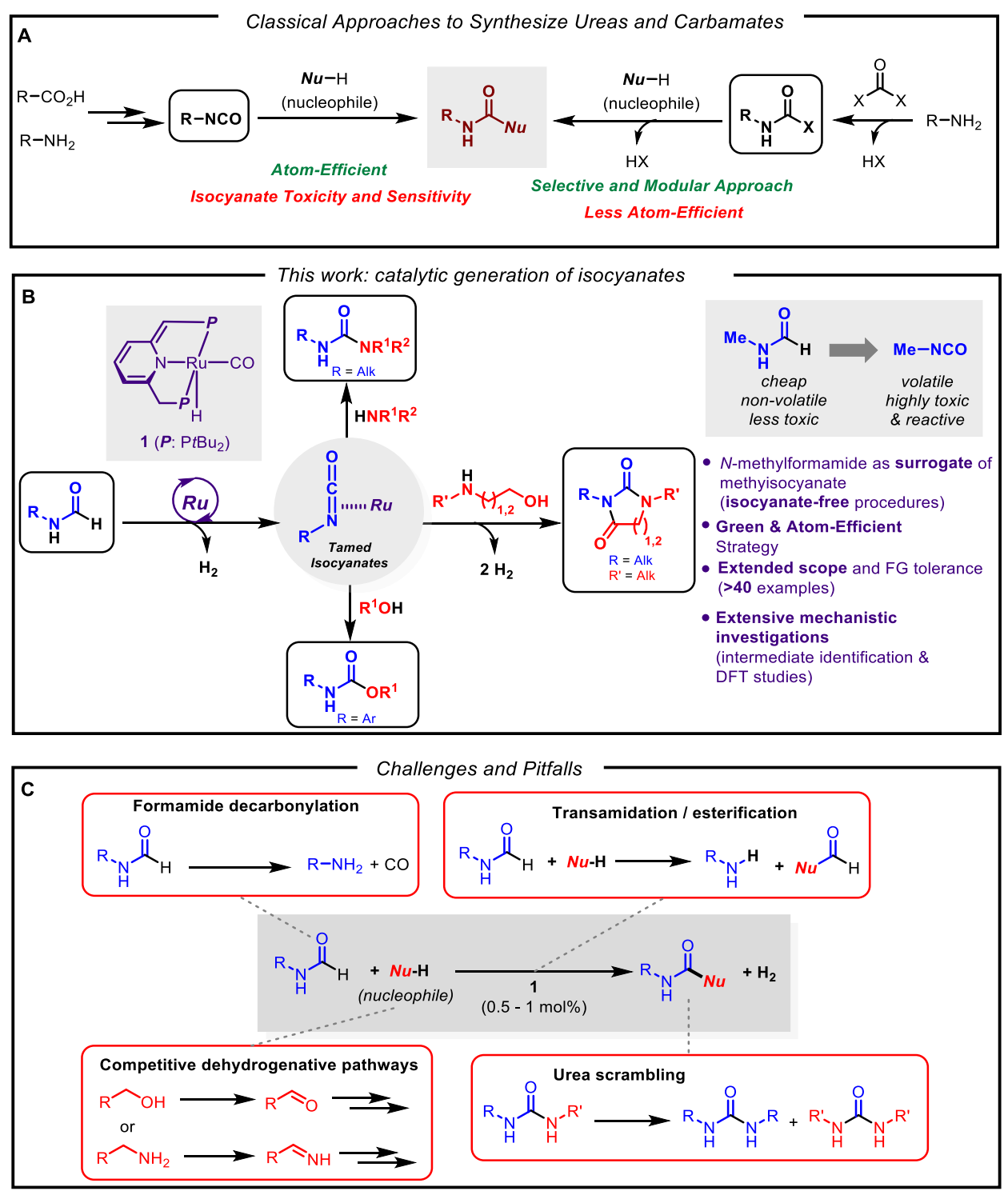

\section{RESULTS AND DISCUSSION}

We have been interested in the behavior of ruthenium(II) and manganese(I) based pincer complexes (for a list of complexes assessed in this study, see Fig. S1 in Supplementary Information) in the activation of covalent $\mathrm{N}-\mathrm{H}$ bonds, such as in amines. ${ }^{20}$ At the outset, we studied the possibility of $\mathrm{N}$ $\mathrm{H}$ activation of formamide derivatives. Indeed, upon reaction with the dearomatized pincer complex (PNP*)RuH(CO) complex $\mathbf{1}^{21}$ (Scheme 2), rapid N-H activation took place, quantitatively providing ruthenium amido complexes in solution (2-5). Remarkably, the formamido complex $\mathbf{2}$ was unstable at higher temperatures $\left(100^{\circ} \mathrm{C}\right)$, releasing hydrogen in an acceptorless fashion, while yielding a novel, crystallographically characterized (Figure 1, left) Ru-isocyanate complex (6). We are unaware of previous reports on isocyanate metal complexes accessed from formamides. This unprecedented result suggested the in situ generation of isocyanic acid (HNCO) via the stoichiometric dehydrogenative decomposition of formamide. Furthermore, the reaction of 
1 with $N$-methyl- or $N$-benzylformamide similarly yielded, respectively, complexes $\mathbf{3}$ and $\mathbf{4}$ in solution at room temperature. Upon heating, these complexes also liberated $\mathrm{H}_{2}$ and were interconverted into the novel isocyanate complexes 7 and $\mathbf{8}$, respectively, featuring new covalent $\mathrm{C}-\mathrm{C}$ bonds between the pincer ligand and the isocyanate moiety, and a hydrogen bond to a formamide molecule (Scheme 2). Complex 7 was crystallographically characterized (Figure 1, middle). The crystallographically characterized isocyanate complex 9 (Figure 1, right), same as 8 except for the hydrogen bonded benzylformamide, was prepared by addition of benzyl isocyanate to 1 . Addition of $\mathrm{H}_{2}$ (2at) to 9 in the presence of excess benzyl isocyanate led to isocyante hydro- genation and formation of $\mathbf{8}$, confirming the structural features of complex 8, as well as the regioselectivity of its binding (covalent binding of the metal complex through the $\mathrm{C}=\mathrm{N}$ bond of the isocyanate moiety). (Scheme 2). This $\mathrm{C}-\mathrm{C}$ covalent binding was further corroborated by NMR, IR and HRMS analyses (see Supporting Information). Interestingly, complex $\mathbf{5}$, formed by the reaction of $N$-methylacetamide with $\mathbf{1}$, did not lead upon heating in solution to any dehydrogenation event. Also, $N, N^{\prime}$-dimethylformamide (DMF) did not react with $\mathbf{1}$ even at elevated temperatures, ruling out a direct oxidative insertion of the formyl $\mathrm{C}-\mathrm{H}$ bond to the metal complex. These initial findings indicate dihydrogen abstraction through metal-ligand cooperation, similarly to the reaction with alcohols (vide infra Scheme 5 and 6). ${ }^{22}$

Scheme 2. Stoichiometric experiments and reactivity towards formamides and isocyanates (NR = no reaction, specified temperatures are bath temperatures).

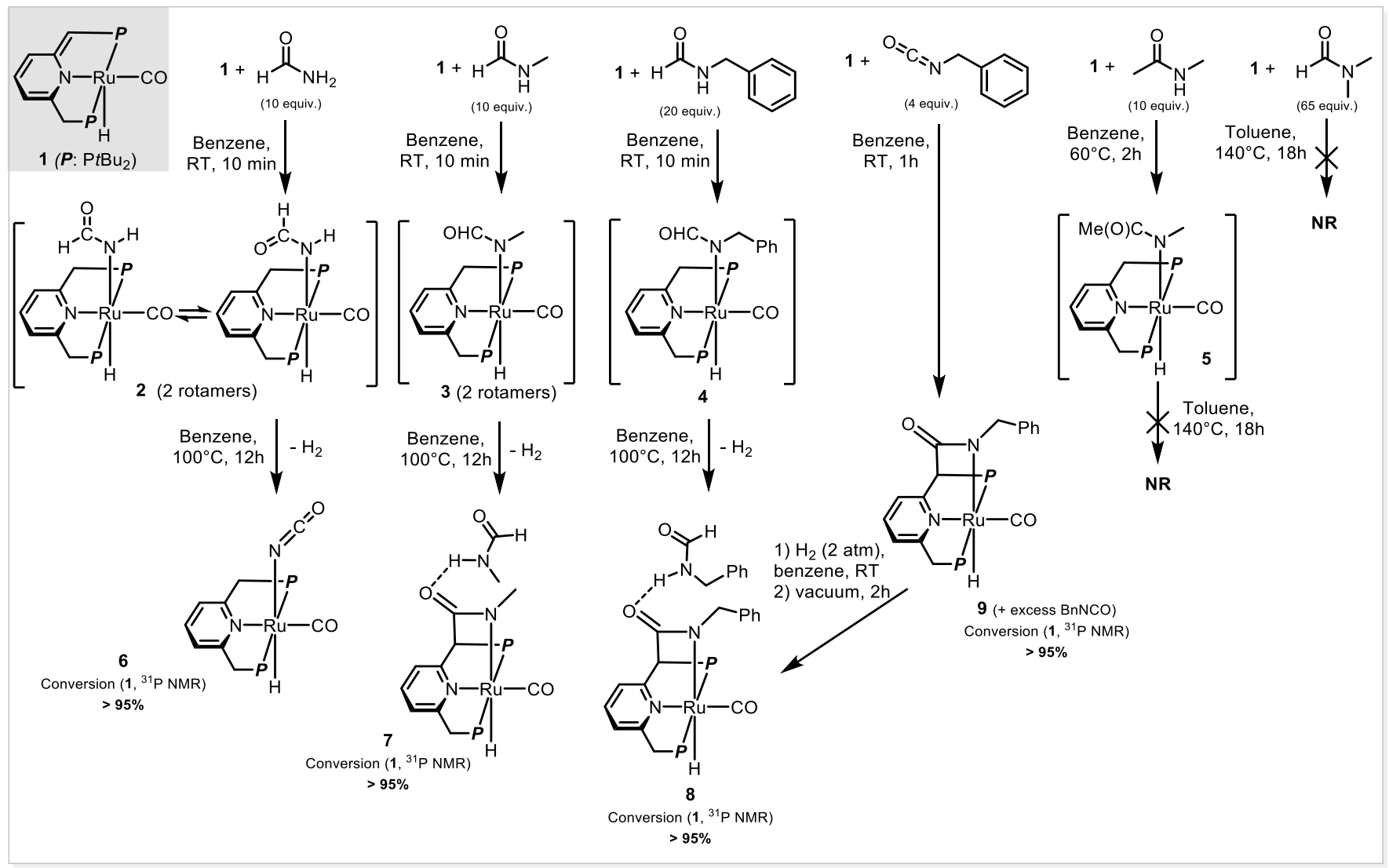




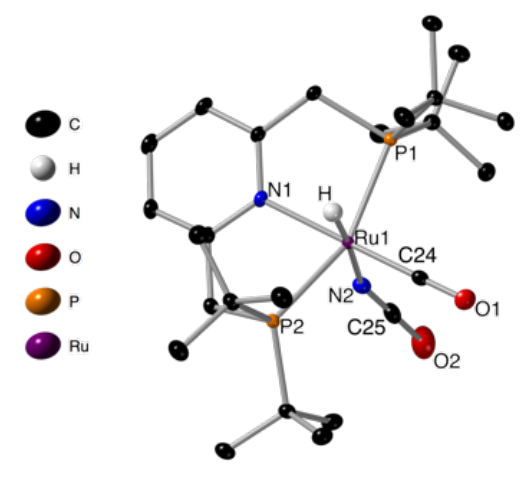

6

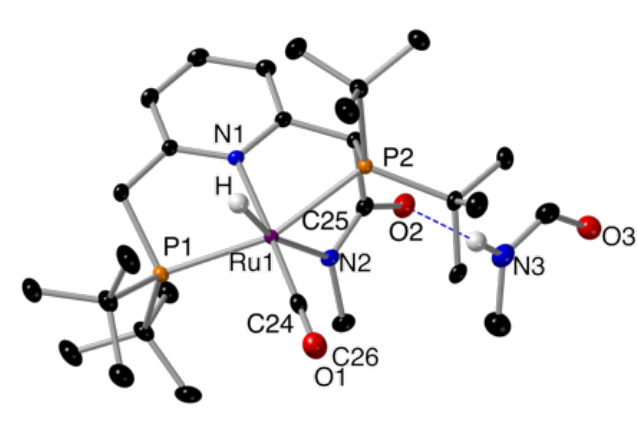

7

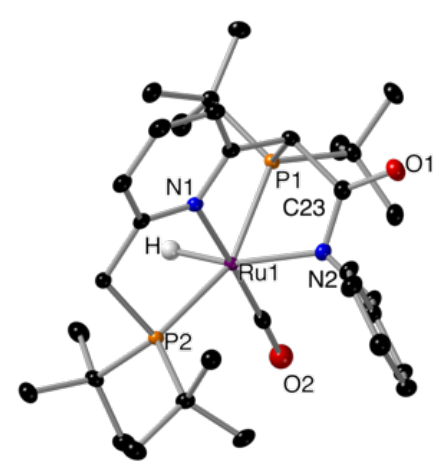

9

Figure 1. X-ray crystal structures of compounds 6, 7 and 9 (thermal ellipsoids drawn at the 50\% probability level, C-H bonds are omitted for clarity). Selected bond distances $(\AA)$ and angles (deg) for 6: Ru(1)-H 1.512(17), Ru(1)-N(1) 2.1518(9), Ru(1)-N(2) 2.2164(11), N(2)-C(25) 1.1503(17), O(2)-C(25) 1.2197(16), C(24)-Ru(1)-N(1) 175.09(4), P(2)-Ru(1)-P(1) 158.270(10), N(2)$\mathrm{Ru}(1)-\mathrm{H}$ 175.2(7), C(25)-N(2)-Ru(1) 151.64(10), N(2)-C(25)-O(2) 178.52(15). For 7: Ru(1)-H 1.51(2), Ru(1)-N(1) 2.1233(11), $\mathrm{Ru}(1)-\mathrm{N}(2)$ 2.2548(11), N(2)-C(25) 1.3210(18), O(2)-C(25) 1.2547(17), C(7)-C(25) 1.5494(19), C(24)-Ru(1)-N(1) 177.28(6), P(1)$\mathrm{Ru}(1)-\mathrm{P}(2)$ 161.444(13), N(2)-Ru(1)-H 164.3(8). For 9: Ru(1)-H 1.505(17), Ru(1)-N(1) 2.1170(10), Ru(1)-N(2) 2.2980(10), N(2)$\mathrm{C}(23)$ 1.3253(15), C(1)-C(23) 1.5512(16), O(1)-C(23) 1.2526(14), P(2)-Ru(1)-P(1) 157.245(11), N(2)-Ru(1)-H 163.5(6), C(31)$\mathrm{Ru}(1)-\mathrm{N}(1)$ 176.61(4), O(1)-C(23)-N(2) 128.05(11).

Based on these experiments, we then turned our attention to devise optimal catalytic protocols. Gratifyingly, in the presence of catalytic amounts of complex 1, monosubstituted formamides could indeed be efficiently and selectively coupled with a large variety of nucleophiles (Scheme 3), including amines, alcohols and aminoalcohols (Scheme 4), exclusively releasing dihydrogen as by-product in an acceptorless fashion. For instance, primary amines were coupled with $\mathrm{N}$-benzylformamide (10a-d) or $\mathrm{N}$-methylformamide $(\mathbf{1 0 e}-\mathbf{m})$ at $100^{\circ} \mathrm{C}$ in 1,2-diethoxyethane as the solvent of choice in the presence of $0.5 \mathrm{~mol} \%$ of 1 under additive-free conditions. To the best of our knowledge, the latter represent the first examples of accessing methyl ureas via the dehydrogenation of formamides, hence successfully surrogating MIC. Our optimization efforts were primarily concerned by the selectivity of this reaction to prevent urea scrambling, which is especially observed at higher temperatures $\left(>120^{\circ} \mathrm{C}\right)$. Additionally, the catalytic coupling of secondary amines was also efficient though exclusively triggered at higher temperatures (typically $135^{\circ} \mathrm{C}$ ). Several experimental observations such as the unsuccessful coupling of various less nucleophilic (e.g. aniline) and/or bulkier amines (e.g. diisopropylamine, even in the presence of the isopropyl version of catalyst $\mathbf{1}$ ) revealed the acute chemoselectivity of complex $\mathbf{1}$ towards different nucleophilic reagents (see Supplementary Information).

\section{Scheme 3. Scope of the catalytic dehydrogenative coupling of monosubstituted formamides with nucleophiles.}



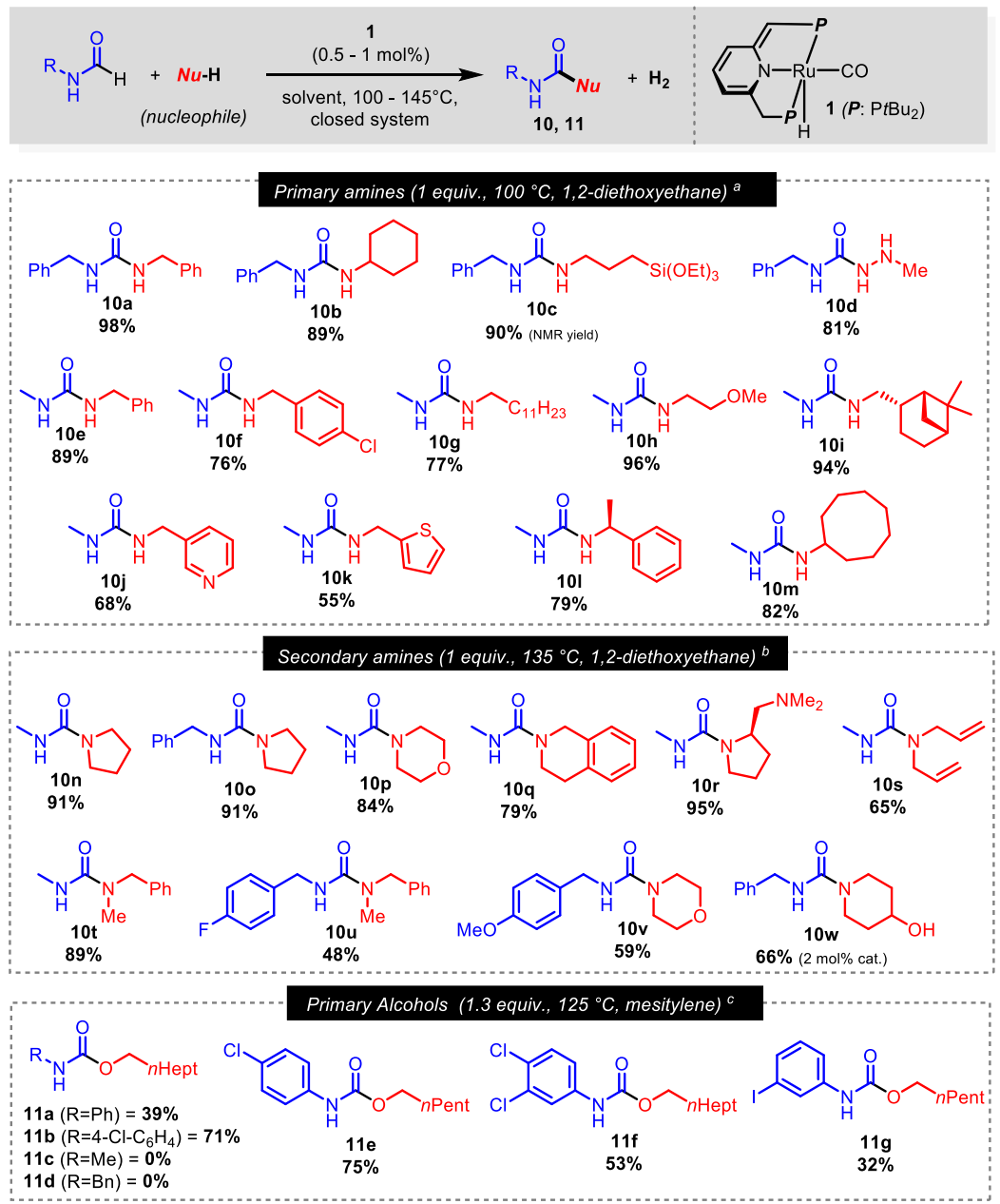
$11 \mathrm{~d}(\mathrm{R}=\mathrm{Bn})=\mathbf{0} \%$
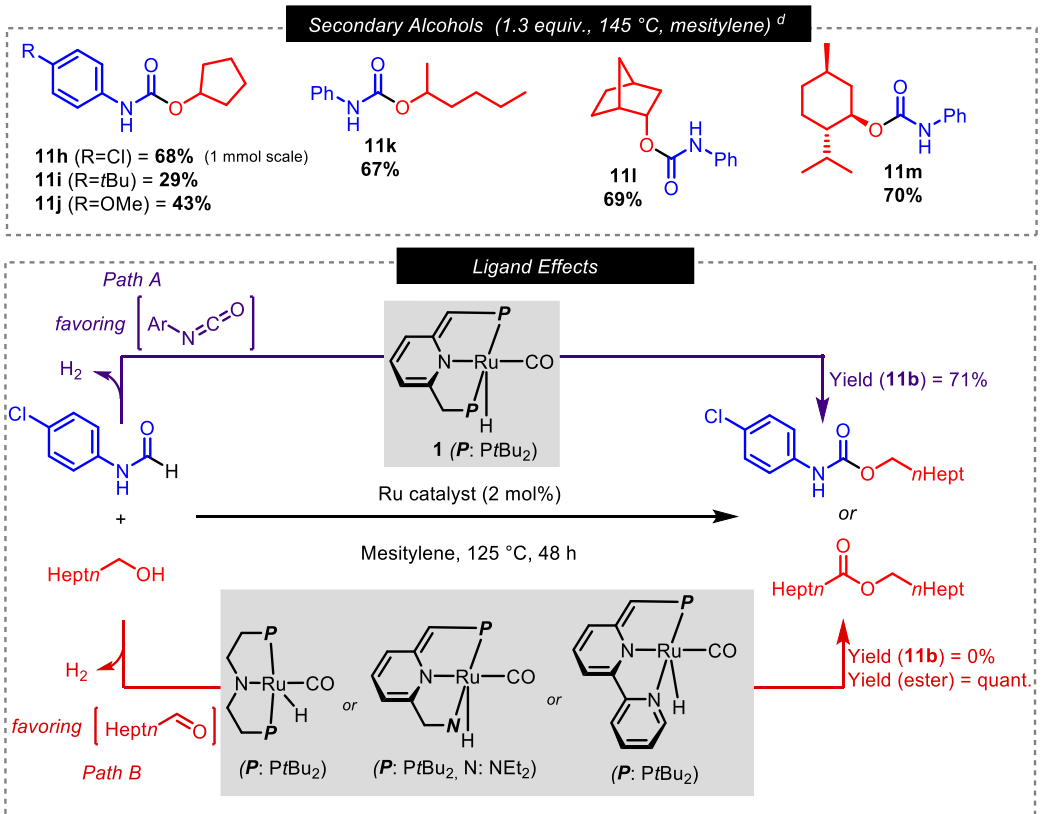

Except if noted otherwise, all yields are reported as isolated yields after column chromatography purification. a: $1 \mathrm{mmol}$ scale, 0.5 mol\% catalytic loading, 1,2-diethoxyethane $(0.5 \mathrm{~mL}), 100^{\circ} \mathrm{C}, 12-20 \mathrm{~h}$. b: $1 \mathrm{mmol}$ scale, $0.5 \mathrm{~mol} \%$ catalytic loading, 1,2 -diethoxyethane $(0.5 \mathrm{~mL}), 135^{\circ} \mathrm{C}, 4-18 \mathrm{~h}$. c: $0.5 \mathrm{mmol}$ scale, $1 \mathrm{~mol} \%$ catalytic loading, mesitylene $(0.5 \mathrm{~mL}), 125^{\circ} \mathrm{C}, 36-48 \mathrm{~h} . \mathrm{d}: 0.5 \mathrm{mmol} \mathrm{scale}, 1 \mathrm{~mol} \%$ catalytic loading, mesitylene $(0.5 \mathrm{~mL}), 145^{\circ} \mathrm{C}, 36-48 \mathrm{~h}$.

The coupling of alcohols with monosubstituted formamides proved more challenging, especially as Ru(II) and Fe(II) pin- cer complexes easily promote competitive alcohol dehydrogenative pathways, limiting the substrate scope to amine 
nucleophiles. ${ }^{16-19}$ We discovered that the choice of the ligand was essential, as complex $\mathbf{1}$ was the only catalyst in our toolbox capable of allowing the selective formation of carbamates (Scheme 3, ligand effects, path $\mathrm{A}$ ), by favoring the dehydrogenation of formamides over that of the primary alcohol (Scheme 3, ligand effects, path B) (see also Supplementary Information). Indeed, primary alcohols could successfully be coupled (11a,b,e-g) with monosubstituted formamides in closed systems at $125^{\circ} \mathrm{C}$ using $1 \mathrm{~mol} \%$ of $\mathbf{1}$. We also noted a strong influence of the electronic effects on the resulting carbamate yield. Thus, more electrophilic $\mathrm{N}$ arylformamides afforded higher yields of the corresponding carbamates, whereas $\mathrm{N}$-alkylformamides $(\mathbf{1 1 b}, \mathbf{c})$ failed to undergo a similar reaction, even at higher temperatures or in the presence of other catalysts. Nonetheless, this methodology could be extended to the coupling of secondary alcohols at $145^{\circ} \mathrm{C}$, albeit in typically moderate yields $(\mathbf{1 1 h}$ $\mathbf{m})$. Moreover, during our investigation, functional groups such as ethers (10h, 10p, 10v and $\mathbf{1 1} \mathbf{j}$ ), tertiary amines (10r), silanes (10c), olefins (10s), unprotected alcohols (10w and 11a-m), halides (10f, 10u, 11b and 11e-h), cyclobutanes (10i), pyridines $(\mathbf{1 0 j})$, thiophenes $(\mathbf{1 0 k})$ and hydrazines (10d) were shown to be tolerated under our reaction conditions. Also, the catalyst did not lead to any scrambling of any potentially sensitive during the synthesis of compounds 10i, 111 and $11 \mathrm{~m}$ ).

In the specific case of amino alcohols (Scheme 4, R'=Alk), a subsequent imido bond- forming reaction ${ }^{23}$ was triggered following the urea synthesis, leading to the synthesis of the 5- membered ring hydantoin derivatives $(\mathbf{1 2 a}, \mathbf{1 2 b}$ and 12f-h) and the 6-membered ring dihydrouracil $(\mathbf{1 2 d}$ and 12i) derivatives. The exceptional temporal selectivity ${ }^{24}$ of this unprecedented transformation led us to access these heterocycles in moderate to excellent overall yields, consequently releasing three equivalents of $\mathrm{H}_{2}$. Although this conceptually new approach could not be extended at this stage to bulkier aminoalcohols (12c) or larger heterocycles (12e), it uniquely demonstrates the unforeseen potential to devise cascade catalytic transformations triggered by formamide dehydrogenation. The current route using formamide dehydrogenative coupling with aminoalcohols may provide an alternative access to core heterocyclic structures of high therapeutic significance; hydantoins ${ }^{25}$ (generally featured in anticonvulsant active ingredients) and uracils ${ }^{26}$ being frequently encountered motifs in drugs.

Scheme 4. Catalytic dehydrogenative coupling of N-alkylformamides with amino alcohols leading to the formation of heterocyclic adducts 12.

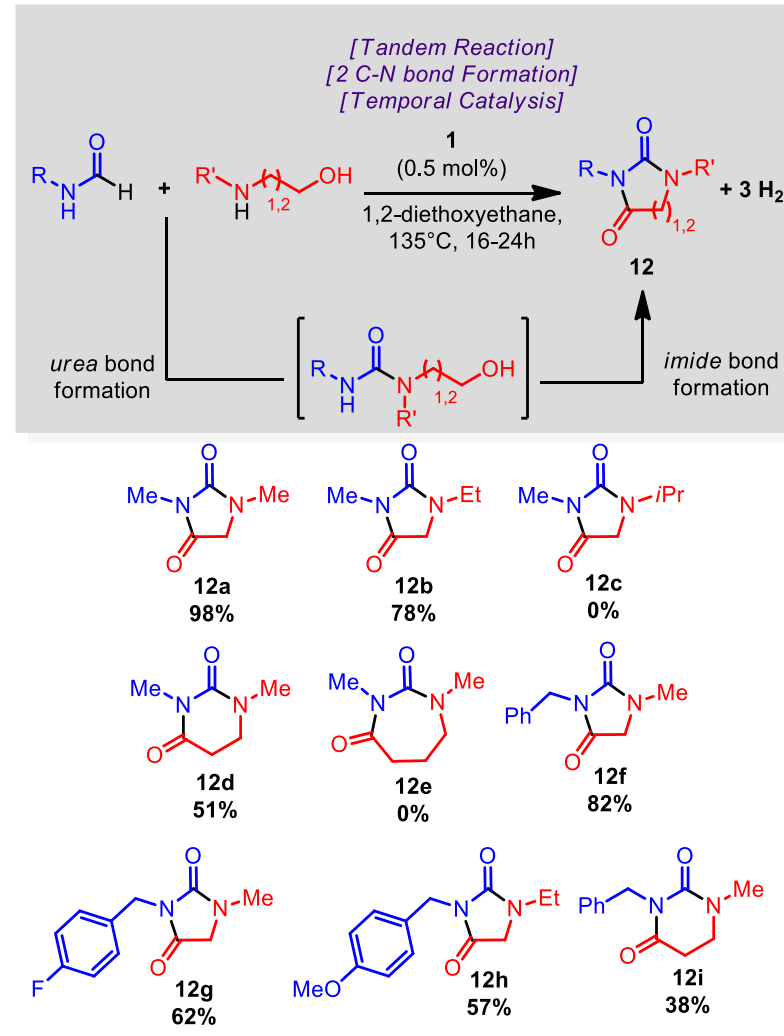

All reactions were carried out on a $1 \mathrm{mmol}$ scale (1:1 equivalents) in 1,2-diethoxyethane ( $0.5 \mathrm{~mL})$ with $0.5 \mathrm{~mol} \%$ of $\mathbf{1}$ in closed systems. All yields are reported as isolated yields after column chromatography purification.

In all the aforementioned cases using catalyst $\mathbf{1}$, it must be stressed that free isocyanates were not detected during the course of these reactions. Given the acute chemoselectivity of complex 1 towards both coupling partners and the absence of observed free isocyanates in solution, a metal-ligand cooperative dehydrogenative coupling mode is indicated (see Scheme 6).

In addition to the insights provided by the stoichiometric control experiments, DFT calculations (see Supporting Information for details) were carried out to further understand the chain of events of these highly-controlled coupling reactions. In agreement with the stoichiometric experiments (see Scheme 2), we can simplify the reaction scheme into two main steps: formamide dehydrogenation (Scheme 5, A) and urea bond formation (Scheme 5, B). In qualitative agreement with the experiments, N-H activation by MLC to form intermediates $\mathbf{I a}$ and $\mathbf{I b}$, and $\mathrm{H}$-abstraction to form transient isocyanate, are readily achieved (TS1 and TS3, not via $\beta$-hydride elimination). Hydrogen release from the dihydride species III might be facilitated through $\mathrm{H}$-bonding (TS4, $\left.\Delta \mathrm{G}^{\ddagger}=33.8 \mathrm{kcal}^{\mathrm{mol}}{ }^{-1}\right) .{ }^{27,28}$ Free isocyanates might be avoided through reversible $\mathrm{C}$ - $\mathrm{C}$ bond formation with the ligand (V via TS5, Scheme 5, B, see also Scheme 2, complex 7). Indeed, the coupling of free isocyanate with $\mathbf{1}$ after $\mathrm{H}_{2}$ loss is downhill in energy by around $15 \mathrm{kcal}^{\mathrm{mol}}{ }^{-1}$. Importantly, this formation of $\mathbf{V}$ (experimentally observed), which can be viewed as a "tamed isocyanate", decreases the electrophilic character of the central isocyanate carbon atom (compared to free methylisocyanate, Scheme 5, C) possibly preventing uncontrolled side-reactions. It also represents the resting state of the catalytic cycles as species $\mathbf{V}$ 
is relatively inert towards nucleophile attack (e.g. benzyl amine, TS6 and Scheme 5, C). In the presence of a nucleophile, the $O$-coordinated intermediate IVb undergoes metalligand cooperative urea bond formation (TS7b, $\Delta \mathrm{G}^{\ddagger}=32.1 \mathrm{kcal}^{\mathrm{mol}}{ }^{-1}$ ). Indeed, the ligand $\mathrm{C}=\mathrm{C}$ double bond is well positioned to deprotonate the incoming nucleophile, while the heterocumulene is activated by the Lewis acidic metal center (see also Scheme 5, C). The benzylic ligand position is then acidic enough to re-protonate the urea-nitrogen, thus releasing the product (TS8).

Scheme 5. DFT calculations of the catalytic coupling of $N$-methylformamide with benzylamine at the $\omega \mathrm{B} 97 \mathrm{X}-\mathrm{V} / \mathrm{def} 2$ TZVP//M06-L GD3/def2-SVP/W06 level of theory.

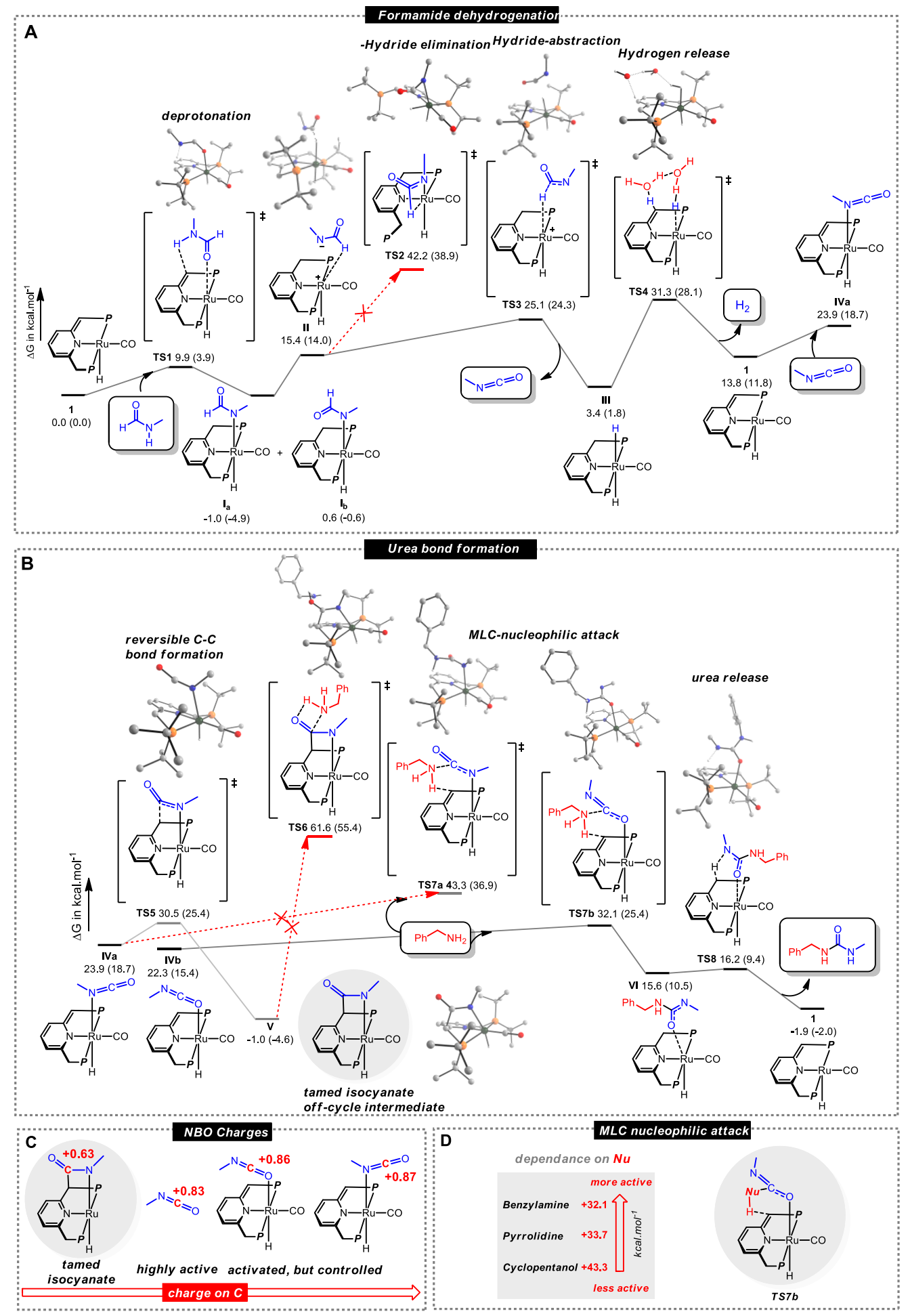


Values correspond to Gibbs free energies in toluene (SMD model) at $298 \mathrm{~K} \mathrm{in} \mathrm{kcal.mol-1}$ with respect to the starting complex 1 $\left(0.0 \mathrm{kcal}^{\mathrm{mol}}{ }^{-1}\right)$. Values in brackets are gas phase Gibbs free energies. DFT-structures with C-H hydrogens, omitted for clarity, are given for key structures and transition states. (A) Reaction potential energy surface for the dehydrogenation of $N$-methylformamide, (B) subsequent urea bond formation, (C) calculated NBO charges on the central carbon atom of different isocyanate moieties and (D) Energies of key transition state TS7b for different substrate nucleophiles.

Although the reaction was computed to be only slightly exergonic $\left(\Delta \mathrm{G}=-1.9 \mathrm{kcal}_{\mathrm{mol}}{ }^{-1}\right)$, the experimentally observed insolubility of the urea product might act as an additional driving force. Importantly, this mechanism also qualitatively accounts for the observed order of reactivity of the different nucleophiles (Scheme 5, D). In order to confirm the resting state character of $\mathbf{V}$, we performed catalytic reactions using complex 7 as the catalyst in the presence of $\mathrm{N}$ benzylformamide and benzylamine, which showed good activity although at slightly higher temperature $\left(130^{\circ} \mathrm{C}\right.$, see SI for additional details). This is in qualitative agreement with the computed mechanism and highlights the reversible nature of the resting state.

\section{Scheme 6. General plausible mechanism (dotted boxes indicate experimentally identified complexes).}

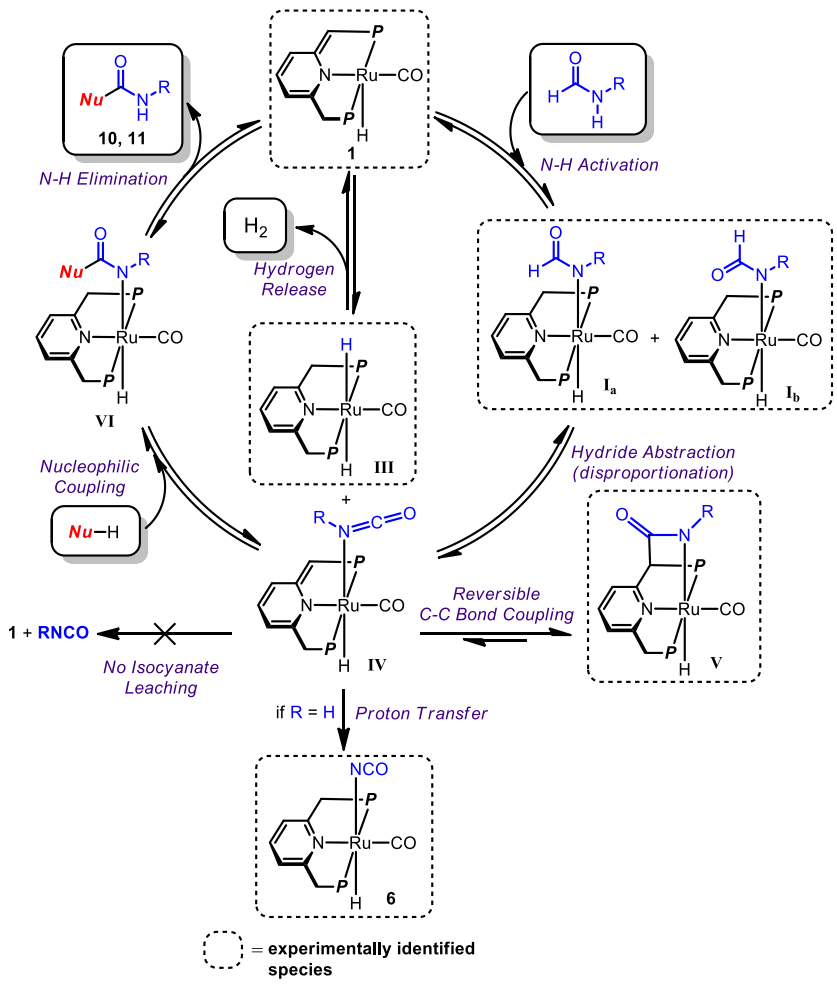

Both our experimental and theoretical studies emphasize the pivotal role of MLC of the pincer ligand of $\mathbf{1}$ by aromatization-dearomatization ${ }^{29}$ on the catalytic activity in almost all fundamental events. In summary, we suggest the following general catalytic mechanism (Scheme 6): (i) rapid N-H activation via MLC of the formamide by $\mathbf{1}$ affords the Ruamido complexes I, (ii) followed by a hydride abstraction, formally a disproportionation reaction, yielding III and presumably IV, (iii) rapid C-C bond activation equilibrium results in adduct $\mathbf{V}$ as the resting state of this catalytic system; (iv) nucleophilic coupling of IV yields VI, this event presumably being the rate determining step; $(v)$ eventually, the catalyst (1) is concomitantly regenerated either through the extrusion of the corresponding urea (10) or carbamate (11), and hydrogen release from the dihydride III.

\section{CONCLUSION}

In conclusion, we report a general and isocyanate-free alternative approach to access valuable unsymmetrical ureas including methyl ureas (avoiding the notorious methylisocyanate), carbamates and the heterocycles hydantoins and dihydrouracils in good yields and excellent selectivities (Scheme 3). Despite the numerous competitive pathways (Scheme 1, C), it was demonstrated that various monosubstituted formamides could be employed as versatile surrogates to isocyanates in generally atom-economical and efficient dehydrogenative coupling reactions. Control experiments, including isolation of key relevant novel complexes (Scheme 2) and DFT studies (Scheme 5) provided critical and unique insights of the mechanisms involved, highlighting the critical ligand's cooperative role. Advantageously, the singular mode of action of complex 1 allows to minimize uncontrolled side-reactions (Scheme 6). Isocyanate-organometallic adducts obtained by dehydrogenation were observed via this approach for the first time (Schemes 2, 6-8), providing structural information on catalytic intermediates (Scheme 6, IV and V). Remarkably, complex 1 also enabled the unprecedented subsequent cascade transformation leading to valuable hydantoin- and dihydrouracil- derivatives (Scheme 4) with an excellent temporal selectivity between the formamide and alcohol dehydrogenation key events. Ultimately, we hope that the fundamental understanding of the catalytic events in play may spark interest in the implementation of such catalytic generation of isocyanates into organic synthesis. We also hope that this work illustrates the need, as well as the opportunities, in seeking surrogates for commonly used hazardous chemicals to establish safer and greener synthetic transformations.

\section{ASSOCIATED CONTENT}

Supporting Information. The Supporting Information is available free of charge via the Internet at http://pubs.acs.org."

Experimental details of synthetic procedures, NMR spectra, X-ray data, and computational details (PDF) Crystallographic data for 6, 7 and 9 (CIF)

\section{AUTHOR INFORMATION}

\section{Corresponding Author}

*david.milstein@weizmann.ac.il

\section{Present Address}

† Laboratoire d'Electrochimie Moléculaire, UMR CNRS 7591, Université Paris Diderot, Sorbonne Paris Cité, 15 rue Jean-Antoine de Baïf, F-75205 Paris Cedex 13, France

Notes

The authors declare no competing interests.

\section{ACKNOWLEDGMENT}


This research was supported by the European Research Council (ERC AdG 692775). D. M. holds the Israel Matz Professorial Chair of Organic Chemistry. J. B. is thankful to the Feinberg graduate school for a postdoctoral fellowship. N. v. W is supported by the Foreign Postdoctoral Fellowship Program of the Israel Academy of Sciences and Humanities.

\section{REFERENCES}

1. Sanderson, K. It's not easy being green. Nature 2011, 469, 1820.

2. Jacobs, M. M.; Mallay, T. F.; Tickner, J. A.; Edwards, S. Alternatives assessment frameworks research needs for the informed substitution of hazardous chemicals. Environ. Health Perspect. 2016, 124, 265-280.

3. Trost, B. M. The atom economy - a search for synthetic efficiency. Science 1991, 254, 1471-1477.

4. Wender, P. A.; Verma V. A.; Paxton, T. J.; Pillow T. H. Functionoriented synthesis, step economy and drug design. Acc. Chem. Res. 2008, 41, 40-49.

5. Newhouse, T.; Baran, P. S.; Hoffmann, R. W. The economies of synthesis. Chem. Soc. Rev. 2009, 38, 3010-3021.

6. Worthy, W. Methyl isocyanate: the chemistry of a hazard. Chem. Eng. News 1985, 63, 27-33.

7. Broughton, E. The Bhopal disaster and its aftermath: a review. Environ. Health 2005, 4, 1-6.

8. Six, C.; Richer, F. "Isocyanates, Organic" in Ulmann's Encyclopedia of Industrial Chemistry (Wiley-VCH, Weinheim, 2012), vol. 20, pp. 63-82.

9. Sheldon, R. A.; Arends, I. W. C. E.; Hanefeld, U. Green chemistry and catalysis (Wiley-VCH Verlag $\mathrm{GmbH} \& \mathrm{Co}$. KGaA, 2007).

10. Khusnutdinova, J. R.; Milstein, D. Metal-ligand cooperation. Angew. Chem. Int. Ed. 2015, 54, 12236-12273.

11. Dobereiner, G. E.; Crabtree, R. H. Dehydrogenation as a substrate-activating strategy in homogeneous transition-metal catalysis. Chem. Rev. 2010, 110, 681-703.

12. Gunanathan, C.; Milstein, D. Applications of acceptorless dehydrogenation and related transformations in chemical synthesis. Science 2013, 341, 249-262.

13. Nguyen, K. D.; Park, B. Y.; Luong, T.; Sato, H.; Garza, V. J.; Krische, M. J. Metal-catalyzed reductive coupling of olefin-derived nucleophiles: reinventing carbonyl addition. Science 2016, 354, 300-306.

14. van der Vlugt, J. I.; Reek, J. N. H. Neutral Tridentate PNP Ligands and Their Hybrid Analogues: Versatile Non-Innocent Scaffolds for Homogeneous Catalysis. Angew. Chem., Int. Ed. 2009, 48, 8832-8846.

15. Kotachi, S.; Tsuji, Y.; Kondo, T.; Watanabe, Y. Ruthenium catalyzed $N, N^{\prime}$-diarylurea synthesis from $N$-aryl substituted formamides and aminoarenes. J. Chem. Soc., Chem. Commun. 1990, 549-550.

16. Kotachi, S.; Kondo, T.; Watanabe, Y. Ruthenium complex-catalyzed synthesis of carbamates by dehydrogenative reaction of formamides with alcohols. Catal. Lett. 1993, 19, 339-343.

17. Kim, S. H.; Hong, S. H. Ruthenium-catalyzed urea synthesis using methanol as the C1 source. Org. Lett. 2016, 18, 212-215 (2016).

18. Krishnakumar, V.; Chatterjee, B.; Gunanathan, C. Rutheniumcatalyzed urea synthesis by $\mathrm{N}-\mathrm{H}$ activation of amines. Inorg. Chem. 2017, 56, 7278-7284.

19. Lane, E. M.; Hazari, N.; Bernskoetter, W. H. Iron-catalyzed urea synthesis: dehydrogenative coupling of methanol and amines. Chem. Sci. 2018, 9, 4003-4008.

20. Khaskin, E.; Iron, M. A.; Shimon, L. J. W.; Zhang, J.; Milstein, D. $\mathrm{N}-\mathrm{H}$ activation of amines and ammonia by $\mathrm{Ru}$ via metal-ligand cooperation. J. Am. Chem. Soc. 2010, 132, 8542-8543.

21. Zhang, J.; Gandelman, M.; Shimon, L. J. W.; Rozenberg, H.; Milstein, D. Electron-rich, bulky ruthenium PNP-type complexes. Acceptorless catalytic alcohol dehydrogenation. Organometallics 2004, 23, 4026-4033.

22. Montag, M.; Zhang, J.; Milstein, D. Aldehyde binding through reversible $\mathrm{C}$-C coupling with the pincer ligand upon alcohol dehydrogenation by a PNP-ruthenium catalyst. J. Am. Chem. Soc. 2012, 134, 10325-10328.

23. Gunanathan, C.; Ben-David, Y.; Milstein, D. Direct synthesis of amides from alcohols and amines from alcohols and amines with liberation of $\mathrm{H}_{2}$. Science 2007, 317, 790-792.

24. Li., L.; Herzon, S. B. Temporal separation of catalytic activities allows anti-Markovnikov reductive functionalization of terminal alkynes. Nat. Chem. 2014, 6, 22-27.

25. Konnert, L.; Lannaty, F.; Martinez, J.; Colacino, E. Recent advances in the synthesis of hydantoins: the state of the art of a valuable scaffold. Chem. Rev. 2017, 117, 13757-13809.

26. Baumann, M.; Baxendale, I. R. An overview of the synthetic routes to the best-selling drugs containing 6-membered heterocycles. Beilstein J. Org. Chem. 2013, 9, 2265-2319.

27. The barrier height for hydrogen release drops from 36 to $33.8 \mathrm{kcal} . \mathrm{mol}^{-1}$ upon addition of a bridging water molecule. Importantly, the water trimer was used as the water source, with one molecule facilitating the $\mathrm{H}_{2}$-release, leaving a residual water dimer.

28. Additional H-bonding with water or formamide is likely to reduce the barrier height further: see Ch'ng, L. C.; Samanta, A. K.; Czako, G.; Bowman, J. M.; Reisler, H. Experimental and theoretical investigations of energy transfer and hydrogen-bond breaking in the water dimer J. Am. Chem. Soc. 2012, 134, 15430-15435.

29. Gunanathan, C.; Milstein, D. Metal-ligand cooperation by aromatization-dearomatization: a new paradigm in bond activation and green catalysis. Acc. Chem. Res. 2011, 44, 588-602.

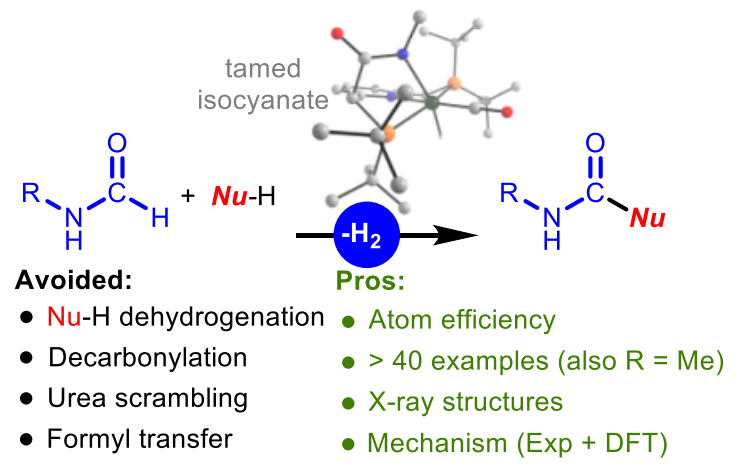

